\title{
POLÍTICAS AMBIENTALES PARA EL CONTROL DE LA CONTAMINACIÓN DIFUSA EN EL REGADÍO
}

\author{
Yolanda Martínez Martínez y José Albiac Murillo \\ Unidad de Economía Agraria. Servicio de Investigación Agroalimentaria. Gobierno de \\ Aragón. Avda. Montañana, 930. 50059 Zaragoza \\ ymartinez@aragob.es.
}

\begin{abstract}
Resumen: La política de control de la contaminación difusa del regadío es una cuestión de interés para la implementación de la Directiva Marco del Agua. En este trabajo se examinan distintas medidas de control de la contaminación por nitratos en el regadío de Flumen-Monegros (Huesca). La evaluación de las políticas se realiza examinando la eficiencia de costes de las medidas, mediante la comparación de los efectos de cada medida sobre la renta de los agricultores y sobre la reducción de la contaminación.
\end{abstract}

Palabras clave: regadío, contaminación por nitratos, medidas de política ambiental.

\begin{abstract}
Non-point source pollution control is an important question for the implementation of the Water Framework Directive. This article analyzes several policy measures to control nitrate pollution in the Flumen-Monegros irrigation district (Huesca). Policy options are evaluated considering the cost-efficiency of measures, by comparing the effects of each measure on farmers net income and pollution abatement.
\end{abstract}

Key words: irrigation, nitrate pollution, environmental policy instruments.

\section{Introducción}

La calidad del agua es un aspecto importante en la gestión de los recursos hídricos. La gestión de estos recursos comprende las tareas de captación, aprovisionamiento y depuración de los recursos hídricos para las actividades humanas, y de pro-

\footnotetext{
* Recibido: 5-12-2002. Aceptado: 4-04-2003.
} 
tección de los ecosistemas y otras funciones ecológicas de los sistemas hídricos. En España, el uso intensivo de los recursos hídricos en las actividades agrarias genera problemas de escasez del recurso, salinización de suelos y aguas, erosión, y contaminación por nutrientes y pesticidas.

En el último siglo, la mecanización de la agricultura y la introducción de variedades de cultivo de alto rendimiento han dado lugar a un fuerte aumento en la utilización de fertilizantes y plaguicidas de origen industrial. Con estas prácticas, los rendimientos agrarios se incrementan, pero las aguas de retorno arrastran parte de las sustancias químicas que constituyen los nutrientes y pesticidas como consecuencia de diversos fenómenos físicos como la escorrentía, la percolación, el lixiviado y la erosión $^{1}$. Estos contaminantes retornan a los cursos de agua por escorrentía o a través de la capa freática y los canales de drenaje de las parcelas, afectando así a la calidad del agua, y por tanto a los diferentes usos posteriores y al estado de los ecosistemas.

La preocupación por los efectos ambientales de las actividades agrícolas ha aumentado en los últimos años. Las actividades agrícolas en regadío tienen una baja rentabilidad en comparación con los usos industriales y urbanos, y reciben fuertes subvenciones tanto a los productos como a la utilización de los recursos hídricos ${ }^{2}$. El precio casi gratuito del agua de regadío genera una demanda de uso agrario ilimitada, y un sistema de racionamiento del recurso mediante concesiones. Las consecuencias son la escasez de recursos hídricos y la degradación del medio ambiente. Estos problemas afectan a la sociedad y a los agricultores, y su solución requiere el establecimiento de una gestión adecuada, sobre todo en zonas áridas y semiáridas donde la escasez de agua genera conflictos entre los usuarios y fuertes impactos ambientales.

Los planteamientos de la economía convencional en la que los factores productivos se reducen a trabajo, tierra y capital, no dan un tratamiento adecuado a los recursos naturales como el agua, y su análisis presenta características específicas. La literatura sobre la economía de los recursos naturales describe ampliamente las características especiales de este tipo de bienes, que además de ser bienes productivos, son también bienes ambientales y activos sociales de gran valor, en los que se basa la sostenibilidad de las actividades humanas.

Las fuentes de contaminación del agua pueden tener un origen localizado espacialmente, como en el caso de las industrias y los vertidos municipales, o bien tener un origen difuso, como sucede en el caso de la agricultura, la construcción, la contaminación de núcleos urbanos, y la que afecta a las explotaciones forestales. La mayoría de los logros en el control de la contaminación se han conseguido para la contaminación de origen localizado. Por el contrario, la contaminación difusa (nonpoint source pollution) es más complicada de resolver, puesto que las herramientas 
desarrolladas para el control de la contaminación localizada no son necesariamente aplicables a la contaminación difusa. Este hecho explica que este tipo de contaminación esté adquiriendo una mayor importancia como responsable de la degradación de los recursos hídricos.

La preocupación por el aporte de nitrógeno procedente de la agricultura y ganadería a las aguas superficiales y subterráneas ha dado lugar a un extenso desarrollo de normas y recomendaciones tendentes a reducir esos aportes. Esta normativa sobre la gestión y la calidad del agua se ha establecido en la Unión Europea a través de la Directiva de Nitratos ${ }^{3}$ y de la reciente Directiva Marco del Agua ${ }^{4}$. La nueva Directiva Marco del Agua supone un cambio en la política europea sobre el agua y extiende la protección a los recursos hídricos superficiales y subterráneos, a todos los ecosistemas acuáticos fluviales de estuarios y aguas marinas conjuntamente, promoviendo la sostenibilidad de los usos y consumos.

Estas cuestiones han provocado un interés creciente en el ámbito de la economía de los recursos naturales, ya que la contaminación de origen agrario está fuertemente ligada a la gestión del agua y a la calidad del medio natural en el que se desarrolla la actividad agrícola. Los estudios empíricos sobre las alternativas de política para el control de este tipo de contaminación, proponen un amplio abanico de instrumentos de control.

Este artículo describe el alcance de los problemas que provoca la contaminación difusa de las actividades de regadío, y sus efectos económicos y ambientales. Así mismo, se presentan algunas conclusiones sobre la eficiencia de costes de diversas medidas de control de la contaminación aplicadas a una zona de regadío en Aragón.

\section{La agricultura como fuente de contaminación del agua}

En la Unión Europea, el uso de fertilizantes químicos experimentó un fuerte incremento en la segunda mitad del siglo veinte, que se ha estabilizado o ha disminuido en las últimas décadas. Existen no obstante grandes diferencias entre los países europeos con respecto a esta evolución. Así, mientras en países como Holanda y Bélgica se siguen utilizando cantidades superiores a los $150 \mathrm{~kg}$ de nitrógeno activo por hectárea, en los países del sur de Europa las cantidades de nitrógeno aplicadas se mantienen por debajo de $100 \mathrm{~kg} / \mathrm{ha}$, si bien partían de valores mucho menores (Cuadro 1).

Paralelamente, el uso de fertilizantes con fósforo ha experimentado en los países del Norte y el Oeste de Europa, una clara tendencia a disminuir, desde $50 \mathrm{~kg} / \mathrm{ha}$ en los años setenta a $20 \mathrm{~kg} / \mathrm{ha}$. En los países del Sur de Europa, el consumo de com- 
puestos de fósforo se ha mantenido relativamente estable en niveles entre 10 y 30 $\mathrm{kg} / \mathrm{ha}$ durante el mismo periodo (MAPA, 1999 y 2000).

En España, la mecanización de la agricultura en los años sesenta y la posterior introducción de variedades de cultivo más productivas implicaron un fuerte aumento en la utilización de fertilizantes minerales. Entre 1960 y 1989, el consumo de nitrógeno pasó de 0,24 a 1,12 millones de toneladas de nitrógeno activo, el de fósforo pasó de 0,36 a 0,53 millones de toneladas de $\mathrm{P}_{2} \mathrm{O}_{5}^{-}$, y el consumo de potasio de 0,07 a 0,37 millones de toneladas de $\mathrm{K}_{2} \mathrm{O}$ (MAPA, 1999 y 2000). En la última década, la tendencia en la utilización media por hectárea de estos fertilizantes continuó creciendo, especialmente en el caso del nitrógeno, con niveles cercanos a la media de la UE (Cuadro 1$)^{5}$.

Cuadro 1. Consumo de fertilizantes comerciales (suma de nitrógeno, fósforo y potasio) en la UE (kg/ha).

\begin{tabular}{|lccccc|}
\hline País & $\mathbf{1 9 9 0}$ & $\mathbf{1 9 9 4}$ & $\mathbf{1 9 9 5}$ & $\mathbf{1 9 9 6}$ & $\mathbf{1 9 9 7}$ \\
Alemania & 276,9 & $\mathbf{1 4 9 , 0}$ & 144,0 & 144,0 & 146,0 \\
Austria & 86,6 & 59,0 & 56,0 & 56,0 & 55,0 \\
Bélgica/Luxemburgo & 257,9 & 227,0 & 219,0 & 222,0 & 228,0 \\
Dinamarca & 227,1 & 167,0 & 157,0 & 158,0 & 160,0 \\
España & $\mathbf{6 4 , 5}$ & $\mathbf{6 3 , 0}$ & $\mathbf{6 0 , 0}$ & $\mathbf{7 1 , 0}$ & $\mathbf{6 7 , 0}$ \\
Finlandia & 173,1 & 138,0 & 122,0 & 110,0 & 111,0 \\
Francia & 187,0 & 154,0 & 161,0 & 166,0 & 166,0 \\
Grecia & 132,0 & 100,0 & 96,0 & 97,0 & 96,0 \\
Países Bajos & 278,4 & 226,0 & 226,0 & 223,0 & 211,0 \\
Irlanda & 152,6 & 109,0 & $\mathbf{1 0 9 , 0}$ & 99,0 & 98,0 \\
Italia & 113,0 & 100,0 & 99,0 & 96,0 & 97,0 \\
Portugal & 70,5 & 62,0 & 63,0 & 65,0 & 60,0 \\
Reino Unido & 128,6 & $\mathbf{1 2 8 , 0}$ & 127,0 & $\mathbf{1 3 5 , 0}$ & 122,0 \\
Suecia & 96,1 & 87,0 & 80,0 & 84,0 & 84,0 \\
Total UE 15 & $\mathbf{1 4 1 , 8}$ & $\mathbf{1 1 6 , 0}$ & $\mathbf{1 1 5 , 0}$ & $\mathbf{1 1 8 , 0}$ & $\mathbf{1 1 6 , 0}$ \\
\hline
\end{tabular}

Fuente: MAPA (2000)

La Agencia Europea del Medio Ambiente (EEA, 1999) señala que la contaminación por nutrientes es la causa principal de la degradación de la calidad del agua en lagos y estuarios, y la segunda causa en los ríos. En el período 1992-1996, alrededor del 65 por ciento de los ríos de la Unión Europea tenían una concentración media anual de nitrógeno que excedía $1 \mathrm{mg} /$ litro de $\mathrm{NO}_{3}-\mathrm{N} .{ }^{6}$ En ese mismo período de tiempo, se detectaron también concentraciones de $33,2 \mathrm{mg} / \mathrm{l} \mathrm{NO}_{3}$ en aproximadamente el 15 por ciento de los casos medidos, y se ha calculado que entre el 50 y el 90 por ciento de 
esta carga de nitrógeno de las aguas superficiales europeas tienen su origen en las actividades agrarias (EEA, 1999) ${ }^{7}$. Las mayores concentraciones se localizan en el Noroeste de Europa, donde la agricultura es especialmente intensiva.

En el caso español, la contaminación por nitratos afecta de forma importante al litoral mediterráneo, con niveles de concentración de nitrato de $500 \mathrm{mg} / 1 \mathrm{NO}_{3}^{-}$en algunas zonas. En grandes áreas de las planas costeras del Júcar (Castellón y Valencia), se superan los $100 \mathrm{mg} / 1 \mathrm{NO}_{3}^{-}$(EEA, 1999; MMA, 2000). También algunas zonas del interior de la península, como la llanura manchega, el aluvial del Ebro y algunos sectores del valle del Guadalquivir presentan niveles entre los 50 y $100 \mathrm{mg} / 1 \mathrm{NO}_{3}^{-}$.

Con respecto a la evolución del consumo de fertilizantes químicos en Aragón, se distingue, tal como se ilustra en la figura 1: un primer período hasta 1989 de crecimiento moderado en el empleo de fertilizantes, con mayor presencia de los fertilizantes nitrogenados, un segundo período hasta 1995 de reducción en el uso de abonos químicos hasta poco más de 58.000 toneladas, y un incremento brusco en los dos últimos años de la serie, con un mayor empleo de fertilizantes potásicos y nitrogenados, superándose las 150.000 toneladas en 1996 y las 200.000 toneladas en $1997 .{ }^{8}$ El consumo de fertilizantes nitrogenados por hectárea en los cultivos de Aragón en 1999 fue de casi $90 \mathrm{~kg} / \mathrm{ha}$.

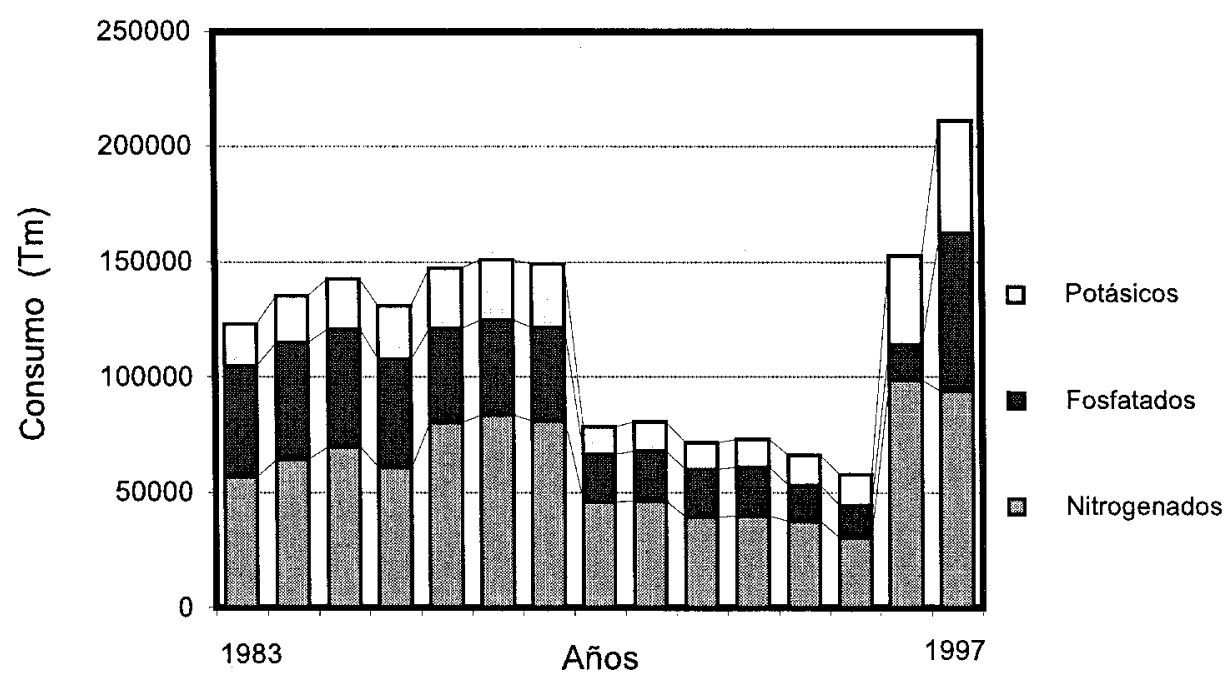

Fuente: DGA (1983 a 1997)

Figura 1. Evolución del consumo de fertilizantes en Aragón (1983-1997). 
Aunque el principal problema en la cuenca media del Ebro es el alto nivel de salinidad, debido al clima árido y a la composición geológica de sus materiales, la contaminación por nitratos constituye un foco de preocupación creciente por tener estrecha vinculación con la actividad de regadio en la zona. Orús et al. (2000) han elaborado una aproximación a las necesidades totales de fertilización nitrogenada en la agricultura de Aragón. Estos cálculos incluyen una valoración tanto de los abonos químicos como de los estiércoles orgánicos, y estiman que la aplicación de fertilizantes puede estar entre un 20 y un 50 por ciento por encima de las necesidades reales de abonado. Las pérdidas de nitrógeno aplicado como fertilizante en forma de nitrato en las aguas de drenaje y escorrentía implican un mal aprovechamiento de un recurso caro, como es el fertilizante nitrogenado.

\section{Efectos negativos de la contaminación por nitratos}

Ribaudo et al. (1999) señalan que los nutrientes del abonado pueden llegar hasta los recursos hídricos por tres vías: por transporte de nutrientes a lo largo de la superficie del suelo por escorrentía superficial, debido al agua de lluvia, nieve o aguas de riego que no penetran en el suelo; por vertido directo de productos químicos al agua superficial, por la existencia de poros o muros fracturados o construcciones mal realizadas; y finalmente por la percolación del agua de lluvia, riego o nieve que produce el lixiviado o lavado de nutrientes a través del suelo.

Los impactos de una carga excesiva de nutrientes pueden manifestarse de forma directa por contaminación del agua potable o como factor de eutrofización que elimina el oxígeno necesario para la supervivencia de los organismos acuáticos. Además, la presencia de altas concentraciones de nitratos en el agua puede tener efectos negativos sobre la salud humana que se han identificado en multitud de estudios. En todos ellos se hace referencia a los riesgos de contraer metahemoglobulemia ${ }^{9}$, sobre todo en niños menores de seis meses de edad, y de cáncer de estómago en adultos con acidez estomacal baja (Keeney y Follet, 1991).

Farré (1998) y Faeth (2000) añaden a los efectos anteriores algunos otros con repercusiones económicas claras, como son la disminución de la transparencia del agua, problemas en el tratamiento por sabor y hedor, con el consiguiente aumento de los costes de depuración del agua para el consumo humano y la producción industrial, y daños en el valor estético y recreativo del entorno acuático.

Las técnicas que pueden emplearse para la depuración del agua contaminada por nitratos son diversas y la decisión sobre cuál escoger depende de la disponibilidad de la tecnología necesaria y del coste de la misma. Las técnicas más habituales son la 
ósmosis inversa, la mezcla con agua más pura, la desnitrificación biológica, el intercambio de iones y la electro-diálisis (Hanley, 1991). Croll y Hayes (1988) plantean con más detalle las ventajas e inconvenientes de cada una y proponen la desnitrificación y el intercambio de iones como las técnicas más adecuadas para la tecnología disponible. La autoridad competente en materia de agua del Reino Unido señala que el intercambio de iones es más barato, y también en Francia se emplea este mismo proceso.

Horner (1975) cuantifica entre 30 y 45 dólares/acre-foot $\left(0,022-0,034 € / \mathrm{m}^{3}\right)$ el coste de depuración mediante la desnitrificación biológica en el valle de San Joaquín (California). De Haen (1982) valora esta técnica en Alemania entre 0,06 y 0,11 mar$\cos / \mathrm{m}^{3}\left(0,03\right.$ y $\left.0,06 € / \mathrm{m}^{3}\right)$, dependiendo de la dimensión de la planta de tratamiento o depuración. En España, el coste de potabilización es de unos $0,15 € / \mathrm{m}^{3}$, y de 0,05 $€ / \mathrm{m}^{3}$ el correspondiente a la eliminación de nitratos.

Para reducir los problemas de contaminación difusa, Ribaudo et al. (1999) han propuesto el uso de prácticas de agricultura ecológica, que utilizan materia orgánica y abonos "verdes" como fertilizantes, y la rotación de cultivos. Estas técnicas favorecen una buena estructura del suelo, disminuyen la erosión y mantienen niveles bajos de nutrientes libres en el suelo, evitando que sean arrastrados hasta los cursos de agua. Además hay que ajustar los aportes de abonos a las necesidades de los cultivos y a las características del suelo de la zona, ya que el exceso de abono no conduce a mejores cosechas, aunque sí reduce el riesgo de estrés de nitrógeno en las plantas.

\section{Normativa sobre control de la contaminación por nitratos}

La normativa europea sobre la gestión y la calidad del agua se ha establecido a través de la Directiva de Nitratos y la Directiva Marco del Agua. La primera de ellas tenía como objetivo reducir la contaminación del agua subterránea causada por los nitratos originados en la actividad agrícola. Esta Directiva imponía a los estados miembros la obligación de identificar las áreas afectadas por este tipo de contaminación o susceptibles de estarlo en un futuro próximo designándolas como "zonas vulnerables". El criterio para identificar estas zonas, era detectar concentraciones de nitrato en el agua próximas o superiores a $50 \mathrm{mg} / \mathrm{NO}_{3}^{-}$. La directiva proponía así mismo la elaboración de "Códigos de Buenas Prácticas Agrarias", en los que se debían detallar tanto el laboreo de la tierra, como el manejo de los cultivos que resultan más favorables para limitar o eliminar la contaminación provocada por la agricultura.

En España, la aplicación de la legislación sobre calidad del agua ha supuesto un avance en el tratamiento de aguas residuales urbanas, pero la Directiva de Nitratos ha llevado un gran retraso, al igual que en otros países comunitarios. Las Comunidades 
Autónomas han establecido con demoras y bajo la amenaza de penalizaciones, los Códigos de Buenas Prácticas Agrarias, las Zonas Vulnerables, los Programas de Acción y las Medidas de Obligado Cumplimiento.

Por su parte, la nueva Directiva Marco del Agua establece: i) una combinación de restricciones de emisiones y estándares de calidad, con fechas limite para que todas las aguas tengan una calidad apropiada; ii) una gestión del agua basada en cuencas y con participación de los usuarios; y iii) un precio del agua a los usuarios que se aproxime al coste completo de recuperación, incluyendo los costes de captación, distribución y tratamiento, los costes medioambientales y los costes del recurso.

Una de las innovaciones de la directiva es el fomento de la gestión de los recursos hídricos por cuencas o unidades hidrológicas y geográficas naturales ${ }^{10}$, con unos precios del agua que incorporen los costes medioambientales, de forma que la gestión de la demanda de agua de riego va a adquirir una gran importancia como consecuencia de la Directiva Marco del Agua.

\section{Análisis económico de la contaminación: una aplicación a la zona de regadío de Flumen-Monegros (Huesca)}

Desde el punto de vista económico, la contaminación difusa es un ejemplo típico de efecto negativo o externalidad negativa de producción, y como se ha indicado la teoría económica señala que la decisión sobre el nivel óptimo de contaminación implica valorar los beneficios y daños de la actividad contaminante para la sociedad. En presencia de externalidades, el valor de los daños no es tenido en cuenta por los agricultores que maximizan su beneficio privado.

La corrección de la externalidad puede llevarse a cabo mediante los distintos instrumentos disponibles, si bien los instrumentos de política que suelen aplicarse generalmente a los problemas de contaminación localizada no son útiles en el caso de la contaminación difusa, ya que las emisiones que produce cada agente no son observables a un coste razonable. En consecuencia los instrumentos óptimos no pueden aplicarse, tal y como han mostrado diversos trabajos empíricos, y por ello el regulador se ve obligado a buscar políticas basadas en medidas sub-óptimas.

La literatura sobre la economía de los recursos naturales clasifica los instrumentos de política para el control de la contaminación siguiendo diversos criterios. En general, puede diferenciarse entre los instrumentos de prevención y los de corrección: los primeros actúan para prevenir la actividad contaminante, mientras que los instru- 
mentos de corrección operan para corregir los daños ya producidos. Así, dentro del primer bloque estarían los instrumentos basados en impuestos ambientales, subvenciones a la reducción de emisiones, regulación sobre niveles de contaminación permitidos, y los mercados de licencias para contaminar. El segundo bloque engloba las medidas de depuración de las aguas contaminadas, o las exigencias de recuperación de zonas contaminadas a los agentes contaminadores.

Para evaluar las medidas de política ambiental, la teoría económica establece como criterio que la medida consiga que el coste marginal coincida con el beneficio marginal de la contaminación, es decir, se establece un nivel de contaminación en el que la última unidad de contaminación tiene un coste igual al beneficio que se obtiene al permitir la contaminación. En términos económicos se sigue un criterio de eficiencia, lo que incluye no sólo determinar la cantidad óptima de contaminación, sino también seleccionar la medida de política ambiental más apropiada para alcanzar la cantidad de contaminación deseada. Las medidas de política ambiental tienen diferentes grados de eficiencia, que dependen de los incentivos que introducen, y suponen distintos costes de administración y control. Ante las dificultades que presenta el control de la contaminación difusa, los autores han sugerido que en algunos casos lo más apropiado es una combinación de los distintos instrumentos disponibles (Hanley et al., 1997).

Los estudios empíricos sobre la contaminación difusa difieren considerablemente tanto en las técnicas empleadas para el análisis como en su complejidad, si bien pueden identificarse dos tipos de estudios que se han realizado más profusamente, y que muestran grandes ventajas en la valoración integrada de los aspectos ambientales y económicos. Un primer tipo incluye los trabajos que incorporan información específica sobre la zona de estudio, y el segundo tipo comprende los estudios que combinan modelos de simulación agronómica con técnicas de programación matemática, que es el caso del ejemplo que se muestra seguidamente.

En el apartado empírico que se presenta a continuación, se muestran algunos resultados obtenidos en el área de regadío de Flumen-Monegros, para el que se analizan los efectos económicos y ambientales de distintas medidas de control de la contaminación. Se ha optado por describir de forma resumida la metodología y las fuentes de datos empleadas, y presentar con mayor detenimiento los resultados sobre las medidas de política ambiental planteadas. El modelo dinámico completo se describe en Martínez (2002).

La construcción de este modelo incluye la simulación, mediante un programa agronómico, de la respuesta del rendimiento de los cultivos del área de estudio ante cambios en las dosis de riego y abonado, así como la obtención de datos de pérdida de agua y contaminación por nitrato. Se han estudiado los cultivos más importantes del área en razón de la superficie que ocupan y de su importancia económica, incorpo- 
rando la información sobre el manejo de los mismos, así como sobre las características de suelo, clima y gestión de los recursos agua y fertilizante nitrogenado de la zona.

El instrumento matemático básico empleado en este análisis es la programación dinámica o teoría del control óptimo, y el modelo utilizado representa el problema de decisión del agricultor sobre las actividades de producción agraria en la zona ligadas a la gestión del agua y a la contaminación. El agricultor maximiza el valor actual de la renta neta de la explotación, y para ello selecciona las asignaciones de fertilizante nitrogenado y de agua de riego a lo largo de un horizonte temporal, sujeto a un conjunto de restricciones agronómicas y de disponibilidad de factores productivos.

La función objetivo del modelo o valor actual de la renta neta de la explotación, se define como la diferencia entre los ingresos originados por la producción de cultivos y los costes de producción, que incluyen los costes fijos y los costes derivados del uso de factores, así como las subvenciones de la PAC. El modelo incorpora también la especificación de funciones de producción y de contaminación por lixiviado para cada cultivo, así como restricciones de mano de obra, agua, sucesión y frecuencia de los cultivos.

Estas funciones de respuesta de los rendimientos y del nitrógeno lixiviado son no lineales, y dependen de los inputs productivos y de la reserva de nitrógeno en el suelo. El modelo incorpora también el proceso dinámico de la reserva de nitrógeno en el suelo, que reproduce las transformaciones físicas del nitrógeno que tienen lugar en el suelo y que dependen de las condiciones ambientales y de la gestión de los inputs.

El modelo se utiliza para simular el escenario actual de precios de los factores y de los productos en la zona de estudio, incluyendo también las ayudas que reciben los productores y los costes de producción. Esta primera simulación permite comparar los valores reales de estas variables con los obtenidos mediante la simulación, de lo que pueden derivarse unas primeras conclusiones sobre el uso y gestión actuales de los factores.

Posteriormente se plantea un conjunto de escenarios de distintas medidas de política ambiental para reducir la contaminación. Todos estos escenarios permiten obtener resultados valiosos para identificar la eficiencia de cada medida de política y sus efectos sobre las rentas de los agricultores y sobre los niveles de contaminación Esta información sirve para evaluar las medidas en función del objetivo que se persigue.

\subsection{Descripción de la zona de estudio}

La superficie estudiada comprende dieciséis municipios de las comarcas de la Hoya de Huesca y Monegros (Huesca), y la zona de regadio tiene una extensión de 
Cuadro 2. Uso de la tierra en el área de estudio (ha).

\begin{tabular}{|lccc|}
\hline & Secano & Regadio & Total región \\
\hline Tierras de cultivo & 14.250 & 45.781 & 60.031 \\
Pradera y pastizal & 1.418 & 0 & 1.418 \\
Terreno forestal & 4.924 & 117 & 5.041 \\
Otras superficies & 11.351 & 0 & 11.351 \\
Superficie total & 31.943 & 45.898 & 77.841 \\
\hline
\end{tabular}

Fuente: DGA (1999)

77.841 ha, de las que 60.000 ha son cultivables y 45.898 ha (el $75 \%)$ son tierras de regadío. El cuadro 2 muestra los distintos usos de la tierra en regadío y secano.

La población de los municipios de la zona de estudio es de unos 11.000 habitantes, y las actividades agrarias tienen una gran importancia. Los cultivos más importantes son los cereales, que ocupan el 45 por ciento de la superficie cultivable y generan el 79 por ciento del valor de la producción agrícola. Los activos agrarios a tiempo total y parcial alcanzan las 1.292 personas, lo que supone el 36 por ciento de la población activa de la zona. El resto de la población activa trabaja en el sector servicios y en el industrial (INE, 1999).

La zona comprende 1.800 explotaciones agrarias, con una superficie media de 20 hectáreas. Este tamaño determina en gran medida el acceso al capital y la rentabilidad de la maquinaria. Cada explotación engloba un número medio de 10 parcelas, cuya dimensión media es de 4 hectáreas, aunque esta medida es muy variable, entre las 0,3 y 13,6 ha, lo que influye en la rentabilidad del uso factores como la maquinaria y la mano de obra.

El clima es semiárido con déficit de agua a lo largo de todo el año, por lo que el riego es esencial en la producción agrícola. Los recursos hídricos de la zona tienen su origen en el embalse de la Sotonera y el Canal del Cinca, y se distribuyen a través del canal de Flumen y el canal de Monegros. Los cultivos de regadío más extendidos son la alfalfa y el maíz, con más de 17.400 hectáreas cada uno (34\%), y los cereales de invierno con $4.177(8 \%)$ y $5.043(10 \%)$ hectáreas para cebada y trigo, respectivamente.

El medio físico que caracteriza la zona ha sido descrito extensamente en el trabajo de Martínez (2002), para el que se elaboró una clasificación de los suelos existentes en el área de acuerdo con la cartografía disponible de los trabajos de Nogués (2000). La clasificación establece cinco tipos de suelo, con una gradación de su potencial productivo, que viene determinada por los parámetros de salinidad, sodicidad, acidez y capacidad de retención de agua, si bien para este ejemplo se ha considerado únicamente el tipo de suelo que tiene una mayor extensión. 
Aunque no existen mediciones de la concentración de nitratos en las aguas de drenaje del polígono de riego del área Flumen-Monegros, los datos sobre calidad del agua que proporciona la Red ICA (Red Integrada de Calidad de Aguas Superficiales) permiten dar una idea aproximada del grado de contaminación que afecta al área.

La figura 2 muestra la evolución de la concentración de nitratos en las aguas del río Flumen a su paso por Sariñena, que puede dar noción de la calidad de los recursos hídricos tras haberse regado la zona de Flumen-Monegros. La serie está constituida por 40 mediciones realizadas dos veces al año (una en el mes de febrero y otra en el mes de septiembre) a lo largo del intervalo entre 1982 y 2002. Esta serie se compara con las correspondientes a las mediciones del río Segre a su paso por Serós (antes de la desembocadura del Cinca) y con las del río Ebro en Mequinenza (antes de la desembocadura del Segre). Las presas de Mequinenza y Ribarroja tienen un efecto de reducción de la concentración de nitratos en el Ebro. La concentración de nitratos en las aguas del Segre ha permanecido siempre por debajo de los $20 \mathrm{mg} / 1 \mathrm{NO}_{3}^{-}$, y únicamente seis de las 20 mediciones superan los $15 \mathrm{mg} / \mathrm{l}$. Estas cifras contrastan con las mediciones correspondientes al Flumen en Sariñena, que se sitúan entre los 20 y los $30 \mathrm{mg} / \mathrm{l}$ e incluso se ha llegado a los $35 \mathrm{mg} / \mathrm{l}$. Los recursos del río Flumen van a parar al río Cinca, y éste es afluente del río Segre, cuyas aguas contribuyen a mejorar la calidad de la masa de agua antes de desembocar en el Ebro, atenuando la baja calidad del agua del Cinca. Como puede apreciarse en la serie de la figura 2, el agua del Ebro en Mequinenza presenta un nivel de contaminación intermedio y en los últimos años su nivel es similar al del río Segre.

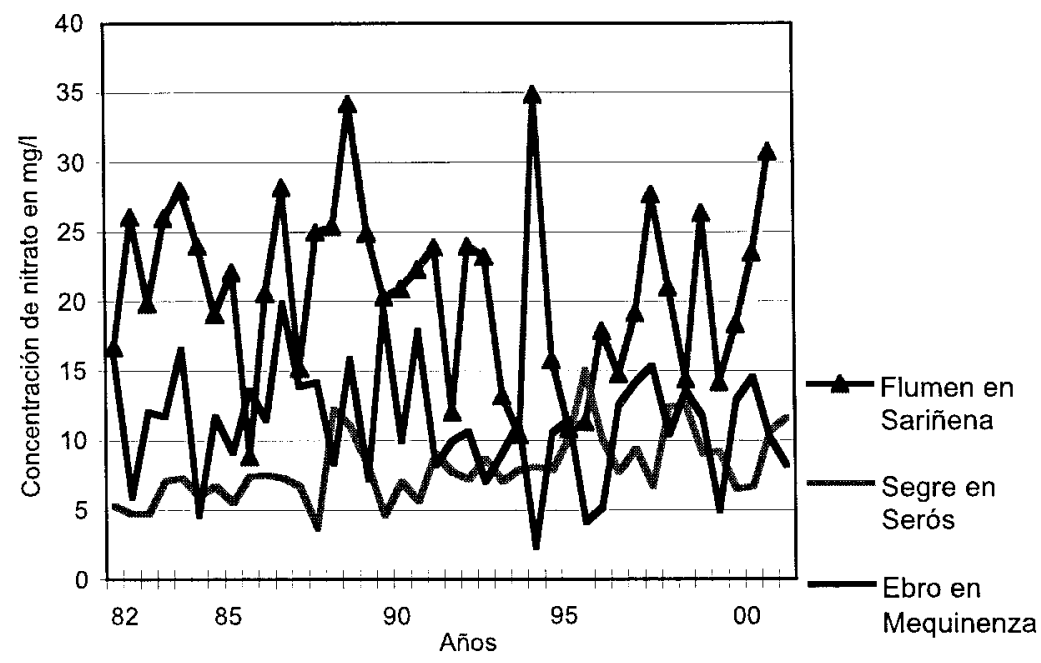

Fuente: CHE (2002)

Figura 2. Evolución de la contaminación por nitratos de 1982 a 2002 en el área de estudio. 


\subsection{Diseño de políticas ambientales}

En este apartado se analizan un conjunto de medidas de control de la contaminación, para determinar la eficiencia de costes de cada medida. Esta ordenación de las medidas de control permite realizar un diseño más apropiado de las politicas ambientales aplicadas a la contaminación difusa. Para evaluar la eficiencia de costes se compara la situación actual o escenario base, con los escenarios correspondientes a cada medida. Las medidas analizadas son el aumento en los precios de los factores productivos agua y nitrógeno, un límite cuantitativo sobre la aplicación de fertilizante, y la introducción de impuestos a las emisiones de lixiviado. El cuadro 3 muestra los resultados de las simulaciones para la región correspondientes a renta neta de la región, uso de inputs productivos, y emisiones contaminantes.

Cuadro 3.Comparación de los resultados en los escenarios simulados para toda la región.

\begin{tabular}{|c|c|c|c|c|c|}
\hline & $\begin{array}{l}\text { Renta neta } \\
(\text { miles } €)\end{array}$ & $\begin{array}{l}\text { Uso de agua } \\
\left(\mathrm{hm}^{3}\right)\end{array}$ & $\begin{array}{l}\text { Uso de nitrógeno } \\
\text { (Tm) }\end{array}$ & $\begin{array}{c}\text { Percolación } \\
\left(\mathrm{hm}^{3}\right)\end{array}$ & $\begin{array}{l}\text { Lixiviado } \\
\text { (Tm) }\end{array}$ \\
\hline Escenario base & 17,1 & 187 & 5.089 & 88 & 1.617 \\
\hline $\begin{array}{l}\text { Escenario } 1 \\
\text { (Precio agua } 0,06 € / \mathrm{m}^{3} \text { ) }\end{array}$ & 11,4 & 139 & 4.949 & 81 & 1.425 \\
\hline $\begin{array}{l}\text { Escenario } 2 \\
\text { (Precio agua } 0,09 € / \mathrm{m}^{3} \text { ) }\end{array}$ & 10,4 & 141 & 6.426 & 144 & 2.335 \\
\hline $\begin{array}{l}\text { Escenario } 3 \\
\text { (Precio nitrógeno } 0,90 € / \mathrm{kg} \text { ) }\end{array}$ & 17,3 & 185 & 4.608 & 97 & 1.369 \\
\hline $\begin{array}{l}\text { Escenario } 4 \\
\text { (Precio nitrógeno } 1,20 € / \mathrm{kg} \text { ) }\end{array}$ & 16,3 & 208 & 4.755 & 129 & 1.284 \\
\hline $\begin{array}{l}\text { Escenario } 5 \\
\text { (Límite N) }\end{array}$ & 16,8 & 171 & 3.991 & 149 & 898 \\
\hline $\begin{array}{l}\text { Escenario } 6 \\
\text { (Tasa emisiones } 0,32 € / \mathrm{kg} \text { ) }\end{array}$ & 16,8 & 184 & 1.470 & 91 & 1.250 \\
\hline $\begin{array}{l}\text { Escenario } 7 \\
\text { (Tasa emisiones } 0,18 € / \mathrm{kg} \text { ) }\end{array}$ & 16,9 & 186 & 4.590 & 95 & 1.470 \\
\hline
\end{tabular}

En el primer escenario se simula un aumento del precio del agua desde 1,2 céntimos de $€ / \mathrm{m}^{3}$ a 6 y 9 céntimos de $€ / \mathrm{m}^{3}$. El incremento del precio del agua reduce la utilización de agua en todos los cultivos, pero al mismo tiempo se produce un cambio en la ocupación de la superficie por cultivos, de forma que el consumo total de agua en la región aumenta por efecto de una mayor ocupación de superficie del maíz y el arroz, que utilizan gran cantidad de agua y nitrógeno. También aumenta el consumo total de nitrógeno en 20 y 192 toneladas, para precios del agua de 6 y 9 céntimos de $€ / \mathrm{m}^{3}$. Este resultado es relevante, pues muestra que un aumento en el precio del agua puede no ser conveniente desde el punto de vista del control de la 
contaminación por nitratos. La demanda global de agua disminuye en 48 y $46 \mathrm{hm}^{3}$, pero la expansión de superficie de los cultivos más intensivos en agua y nitrógeno puede suponer un aumento del lixiviado y de la percolación, como ocurre cuando el precio es de 9 céntimos de $€ / \mathrm{m}^{3}$ (escenario 2). El coste de esta medida para los agricultores de la zona es alto, ya que las pérdidas de renta neta están entre los 570.000 y $\operatorname{los} 670.000$ euros, para 6 y 9 céntimos de $€ / \mathrm{m}^{3}$ respectivamente.

Al aumentar el precio del nitrógeno hasta $0,9 € / \mathrm{kg}$ (escenario 3), la utilización de nitrógeno se reduce ligeramente $(-16 \%)$, y en consecuencia el lixiviado de nitrógeno cae en $250 \mathrm{Tm}(-15 \%)$. En el caso de un aumento del precio del factor nitrógeno hasta $1,2 € / \mathrm{kg}$ (escenario 4), los efectos sobre la utilización de inputs y sobre la contaminación de la región están ligados tanto a los cambios de intensidad del factor nitrógeno, como a los cambios de superficie ocupada por cada cultivo. El aumento del precio del nitrógeno provoca una disminución en el consumo de nitrógeno de todos los cultivos, pero el efecto sobre la utilización global está influido al igual que en el primer escenario por los cambios en la distribución de superficie. El coste para los agricultores en términos de renta neta del escenario de precio $1,2 € / \mathrm{kg}$ es menor que el escenario anterior, con una pérdida de margen neto del 4 por cien y una disminución en el lixiviado de $333 \mathrm{Tm}$ (-21\%).

El quinto escenario introduce un límite en el uso de nitrógeno en las actividades de cultivo, lo que supone una utilización de nitrógeno activo en la zona de 3.991 toneladas, con una reducción del 22 por cien. El límite establecido es de $250 \mathrm{~kg} / \mathrm{ha}$ para el maíz, $100 \mathrm{~kg} / \mathrm{ha}$ para los cereales de invierno y para el arroz, y $50 \mathrm{~kg} / \mathrm{ha}$ para el girasol y la alfalfa. Este instrumento es similar al que tratan de introducir los códigos de buenas prácticas agrarias, establecidos por la Directiva de Nitratos europea, y tiene la ventaja de que el coste para los agricultores de la zona es inferior a los del resto de escenarios planteados, pues el lixiviado se reduce en 719 toneladas anuales $(-44 \%)$ con una reducción de la renta neta de 300.000 euros (-2\%). Por tanto, esta medida es preferible desde el punto de vista social y para los agricultor a otras con efectos negativos sensiblemente mayores sobre el margen neto (Figura 3).

Algunos trabajos en los que se propone un límite cuantitativo, como los de Mapp et al. (1994) en Estados Unidos, y Schmid (2001) en Austria, obtienen unos costes de reducción del lixiviado algo mayores que los calculados en este estudio. Esta solución de limitar el abonado es la que se recomienda en multitud de trabajos, como los de Knickel (1998), Miettinen (1998) y Weinberg y Wilen (2000), y es coherente con los resultados de Martínez et al. (2001), que estiman un coste moderado, cercano al 4 por cien de la renta neta en la misma zona de estudio, para conseguir una reducción del 40 por ciento en el lixiviado.

El último escenario considerado es la introducción de un impuesto directo sobre las emisiones contaminantes, igual al coste de depuración del nitrato presente en el 

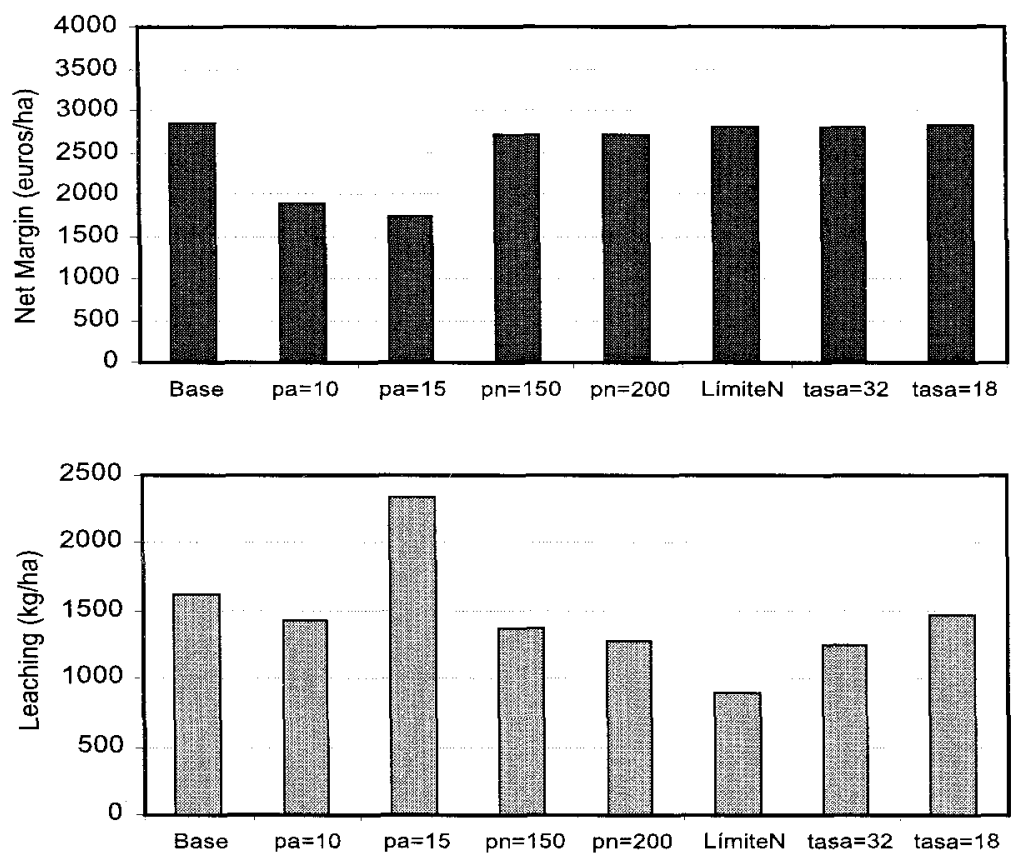

Figura 3. Resultados de renta neta y lixiviado en los escenarios simulados.

agua. Un impuesto directo produce una fuerte reducción de lixiviado, ya que un impuesto sobre las emisiones igual a $0,32 € / \mathrm{kg}$ (escenario 6), hace caer en $3.619 \mathrm{Tm}$ la demanda de abono nitrogenado, y reduce en $367 \mathrm{Tm}$ las emisiones contaminantes $(-22 \%)$. La ocupación de superficie no se modifica, y el coste de esta medida para toda la zona es de 270.000 euros al año $(-2 \%)$.

La introducción de un impuesto menor sobre el lixiviado de $0,18 € / \mathrm{kg}$ (escenario 7 ), provoca una caída de $500 \mathrm{Tm}$ en la demanda de abono nitrogenado con respecto al escenario base, y las emisiones de nitrógeno disminuyen en $147 \mathrm{Tm}$ (-10\%). La ocupación por cultivo se mantiene con respecto a la situación inicial y la renta neta cae en casi 200.000 euros $(-5 \%)$.

El problema de introducir un impuesto sobre las emisiones contaminantes es la dificultad y elevado coste que tiene la observación de la cantidad de emisiones de cada explotación. La alternativa de implantar un limite cuantitativo a las dosis de fertilizante tiene como principal obstáculo el poder controlar que los agricultores cumplen efectivamente tales límites. La introducción de un sistema de ayudas económicas dirigidas a aque- 
llos agricultores que se sujeten a un sistema de aplicación controlada de las dosis de abonado, podría resolver este problema. El control podrían realizarlo las comunidades de regantes mediante verificaciones en el abonado y mediciones en los canales de drenaje.

\section{Conclusiones}

Los efectos de distintas medidas de control de la contaminación por nitrógeno, se han examinado mediante un modelo regional de las seis actividades de cultivo más importantes en el regadío de Flumen-Monegros. La comparación de los distintos escenarios que representan la aplicación de cada medida, permite establecer una jerarquía entre las medidas. La medida más coste-eficiente es la fijación de límites cuantitativos sobre las dosis de fertilizante nitrogenado, seguida por la introducción de un impuesto sobre las emisiones contaminantes de lixiviado de nitrógeno, mientras que las medidas menos coste-eficientes son los aumentos en el precio del agua o en el precio del fertilizante.

Los efectos de las medidas de control dependen por una parte de los cambios en la intensidad del uso de los factores agua y nitrógeno en cada cultivo, y por otra de los cambios en la superficie ocupada por cada cultivo o sustitución entre cultivos. Así por ejemplo, un aumento en el precio del agua de riego reduce el lixiviado por hectárea en todos los cultivos de forma individual, pero conduce a un incremento en el nivel de emisiones en toda la región como consecuencia del incremento de superficie ocupada por los cultivos más intensivos en fertilizante nitrogenado y en emisiones de nitrógeno. Este trabajo incorpora ambos efectos en la evaluación de las medidas de control de contaminación por nitratos. La evaluación sirve para ordenar las medidas según su eficiencia de costes, una información relevante para los responsables de la toma de decisiones en el control de la contaminación por nitratos. Los resultados están en concordancia con la literatura internacional, e indican que el establecimiento de límites en la cantidad de fertilizante nitrogenado es la medida que tiene una mayor eficiencia de costes.

Una cuestión importante en el establecimiento de instrumentos para reducir la contaminación, es la implementación de las medidas y sus costes de control, que determinan la eficiencia final de los instrumentos. Las medidas más costosas de administrar y controlar son precisamente las más eficientes, por lo que el agente responsable del control de la contaminación puede verse obligado a elegir una medida más fácil de administrar, aunque no sea la más coste-eficiente. Medidas alternativas como la introducción del "doctor ambiental" en la que el agricultor puede elegir entre un precio de fertilizante elevado o un especialista que realiza el abonado, o la introducción de impuestos sobre el uso de la tierra (land use tax) según el tipo de cultivo pueden ser preferibles a las óptimas, por su menor dificultad de gestión y control y sus menores costes de administración. 
Por último es necesario resaltar la importancia del control periódico sobre la efectividad y el cumplimiento de las medidas, ya que los efectos ambientales pueden variar en el tiempo y en el espacio, y ello puede requerir una revisión de los instrumentos establecidos para adecuarlos al nuevo contexto.

\section{Notas}

1 La percolación es la infiltración del agua hacia la capa freática. En este movimiento hacia zonas del suelo más profundas, el agua puede arrastrar importantes cantidades de nitrógeno disuelto y sales, que posteriormente alcanzan las aguas de los ríos por los conductos de drenaje. La lixiviación es el fenómeno de lavado de nutrientes y sales por la percolación del agua que es absorbida por el suelo. La contaminación por fósforo está ligada a la erosión del suelo.

$2 \mathrm{El}$ precio medio del agua en el sector terciario es $0,7 € / \mathrm{m}^{3}$ frente a un precio medio de $0,02 € / \mathrm{m}^{3}$ en el sector agrario (INE, 2001).

3 Directiva del Consejo (91/676/CEE).

4 Directiva del Consejo (2000/49/CEE).

5 En España, la aplicación media de fertilizantes minerales es de $68 \mathrm{~kg} / \mathrm{ha}$ para el nitrógeno, $34 \mathrm{~kg} / \mathrm{ha}$ para el fósforo, y $29 \mathrm{~kg} / \mathrm{ha}$ para el potasio, mientras que en la UE la media es de unos $70 \mathrm{~kg} / \mathrm{ha}, 25 \mathrm{~kg} / \mathrm{ha}$, y $30 \mathrm{~kg} / \mathrm{ha}$, respectivamente (MAPA, 1999 y 2000 ).

$6 \mathrm{La}$ expresión $\mathrm{NO}_{3}-\mathrm{N}$ indica el contenido de nitrógeno $(\mathrm{N})$ en el ión nitrato $\left(\mathrm{NO}_{3}^{-}\right)$, de forma que $1 \mathrm{mg}$ de $\mathrm{NO}_{3}$ - $\mathrm{N}$ está contenido en 4,43 $\mathrm{mg}$ de nitrato (es decir, en 4,43 $\mathrm{mg}$ de nitrato hay $1 \mathrm{mg}$ de nitrógeno).

7 Estos porcentajes varian dependiendo de los países, tal y como se muestra en FAO (1997).

8 El comportamiento de la serie entre 1990 y 1995 parece un poco extraño. La caída en el abonado puede explicarse en parte por algún año de sequía, pero puede haber problemas de elaboración estadística.

9 Consiste en la presencia de un exceso de metahemoglobina en la sangre, que dificulta el transporte de oxígeno.

10 Las experiencias de gestión con la cooperación de varios países llevadas a cabo en las cuencas del Rhin, Elba o Schelde, son ejemplos de los resultados positivos logrados mediante la gestión por cuencas.

\section{Bibliografía}

Confederación Hidrográfica del Ebro De Haen, H. (1982): Economic aspects of (2002): Series de datos de calidad de aguas. CHE. Zaragoza, Ministerio de Medio Ambiente. Datos disponibles en www.chebro.es. policies to control nitrate contamination resulting from agricultural production. European Review of Agricultural Economics, 9, p. 443-465. 
Diputación General de Aragón (1993-1997): Macromagnitudes del Sector Agrario Aragonés. 1993-97. Zaragoza, Diputación General de Aragón. Departamento de Agricultura y Medio Ambiente.

Diputación General de Aragón (1999): Base de datos $1 T$ de superficie de cultivos por término municipal para Aragón 1987-99. Zaragoza, Diputación General de Aragón. Departamento de Agricultura.

Environmental European Agency (1999): Nutrients in European Ecosystems. Environmental assessment report, 4 .

FAO (Food and Agricultural Organization of the United Nations) (1997): Lucha contra la contaminación agricola de los recursos bidricos. Estudios FAO Riego y Drenaje, 55. http://www.fao.org/.

Farré, I. (1998): Respuesta del Maíz (Zea mays L.) y Sorgo (Sorghum bicolor L. Moench) al Riego Deficitario, Agronomia y Modelización. Lérida, Universidad de Lérida, Escuela Técnica Superior de Ingeniería Agraria (tesis doctoral).

Feather, P. y Hellerstein, D. (1997): Calibrating Benefit Function Transfer to Assess the Conservattion Reserve Program. American Journal of Agricultural Economics, 79 (1), p. $151-162$.

Hanley, N. (1997): The Economics of Nitrate Pollution Control in the UK. En Harley, N., Shogren, J. y White, B., Environmental Economics in Theory and Practice. Oxford University Press. Capítulo 6, p. 91-116.

Hanley, N., Shogren, J. y White, B. (1997): Environmental Economics in Theory and Practice. Oxford University Press.
Horner G. (1975): Internalizing agricultural nitrogen pollution externalities: a case study. American Journal of Agricultural Economics, 57, p. 33-39.

Instituto Nacional de Estadística (2001): Estadísticas del Agua en España 1999. Madrid, INE.

Keeney, D.R. y Follet, R.F. (1991): Managing nitrogen for groundwater quality and farm profitability: overview and introduction. En Follet, R.F., Keeney, D.R. y Cruse, R.M. (eds.) Managing nitrogen for groundwater quality and farm profitability. Madison, Wisconsin, Soil Science Society of America Inc., p. 1-7.

Knickel, K. (1998): First Results of Model Calculations. En: Gazzola, L. y Roest, K. (eds.), Economic Instruments for Nitrogen Control in European Agriculture. Reggio Emilia, Research Center on Animal Production.

Mapp, H., Bernardo, D., Sabbagh, S., Geleta, S. y Watkins, K. (1994): Economic and environmental impacts of limiting nitrogen use to Project water quality: A stochastic regional analysis. American Journal of Agricultural Economics, 76 (4), p. 889-903.

Martínez, Y., Uku, S. y Albiac, J. (2001): La Calidad del Agua y la Gestión del regadío. Economia Agraria y Recursos Naturales. AEEA (en prensa).

Martínez, Y. (2002): Análisis económico y ambiental de la contaminación por nitratos en el regadio. Zaragoza Universidad de Zaragoza (tesis doctoral).

Miettinen, A. (1993): The Effectiveness and Feasibility of Economic Incentives of Input Control in the Mitigation of 
Agricultural Water Pollution. Agricultural Science in Finland, 2, p. 453-464.

Ministerio de Agricultura, Pesca y Alimentación (1999): Anuario de Estadística Agraria. Madrid, MAPA.

Ministerio de Agricultura, Pesca y Alimentación (2000): Hechos y Cifras del Sector Agroalimentario Español 2000. Madrid, MAPA, Secretaría General Técnica.

Ministerio de Medio Ambiente (2000): Libro Blanco del Agua en España. Madrid, MMA, Secretaría General Técnica.

Nogués, J. (2000): Tipos de suelo y eficiencia de riego en la zona de Flumen-Monegros. Zaragoza.

Orús, F., Quilez, D. y Betrán, J. (2000): El Código de Buenas Prácticas Agrarias (I). Fertilización Nitrogenada y Contaminación por Nitratos. Zaragoza, Diputación General de Aragón. Dirección General de
Tecnología Agraria, Informaciones Técnicas n. ${ }^{\circ} 93$.

Ribaudo, M., Horan, R. y Smith, M. (1999): Economics of Water Quality Protection From Nonpoint Sources. Agricultural Economic Report, 782.

Schmid, E. (2001): Efficient Policy Design to Control Effluents from Agriculture. Viena, Universidad de Ciencias Agrarias (tesis cloctoral).

Silvander, U. y Drake, L. (1989): Economic loss in fisheries and aquaculture by agricultural nitrogen leakage. En: Dubgaard, A. y Nielsen, A. (eds.) Economic Aspects of Environmental Regulation in Agriculture. Kiel, Wissenschaftsverlag Vauk Kiel.

Weinberg, M. y Wilen, J.E. (2000): Efficiency Benefits versus Transaction Costs in Nonpoint Source Pollution Control. En VVAA, $8 b t$ Annual Conference of the european of the European Association of Environmental and Resource Economists. Tilburg (Holanda). 